\title{
Estilos de afrontamiento en pacientes con cáncer de cuello uterino. Servicio de ginecología de un instituto nacional
}

\author{
Baiocchi-Morales Antonet ${ }^{1}$, Lugo-Ostos Evila ${ }^{1}$, Palomino-Sanchez Natali' ${ }^{1}$ Tam-Phun Elena ${ }^{2}$
}

\section{RESUMEN}

Objetivo: determinar los estilos de afrontamiento en las pacientes con cáncer de cuello uterino. Material y métodos: estudio descriptivo de corte transversal. La muestra fue de 150 pacientes en un hospital nacional de enfermedades neoplásicas. Para la recolección de datos se utilizó un cuestionario validado, estructurado con 30 preguntas relacionadas con estilos de afrontamiento: problema, emoción, adicionales. El inventario se sometió a una prueba piloto, obteniendo la confiabilidad de á $=0,73$. Los datos fueron recolectados mediante la entrevista con previo consentimiento informado, luego, los datos se analizaron porcentualmente en el programa SPSS versión 17. Resultados: el estilo positivo más empleado enfocado a la emoción fue «siempre»: busco la ayuda de Dios, el 87\%; enfocado al problema fue «a menudo»: elaboró un plan de acción, el 73,3\%; el estilo adicional: me vuelco en el trabajo y en otras actividades sustitutivas para apartar cosas de mi mente, el $65 \%$. Los estilos de afrontamiento adicionales negativos más empleado fue «a veces»: reduzco la cantidad de esfuerzo que pongo en marcha para resolver el problema, el 79\%; enfocado a la emoción: siento mucho malestar emocional y termino por expresar ese malestar, el 70\%; enfocado al problema «a menudo»: me obligo a esperar el momento adecuado para hacer algo, el 53,3\%. Conclusiones: los estilos de afrontamiento más empleados por las pacientes fueron los estilos positivos y enfocados a la emoción.

Palabras clave: cáncer de cuello uterino, ginecología. Perú. (Fuente DeCs BIREME).

\section{Coping styles in patients with cervical cancer. Gynecology service of a National Institute}

\begin{abstract}
Objetive: Determine coping styles of patients with cervical cancer. Material and methods: Cross-sectional descriptive study. The sample was 150 patients in a national hospital of neoplastic diseases. For data collection we use a validated questionnaire, it was structured with 30 questions related to coping styles as: problem, emotion, additional, the inventory was underwent by a pilot test, obtaining the reliability of á $=0.73$. Data were collected by interview with informed consent, after that, data were analized by percentage terms in the program SPSS version 17. Results: The most used positive style focused on emotion was «always»: I look God's help 87\%, focused on the problem was «often»: I make a plan of action $73.3 \%$, the additional style: I turn to work or another substitute activities to take my mind of things. $65 \%$. The additional negative coping style most often used «sometimes» was: I reduce the amount of effort which I'm putting into solving the problem 79\%, focused on the emotion: I feel a lot of emotional distress and I find myself expressing those feelings a lot $70 \%$, focused on the problem «often»: I force myself to wait for the right time to do something 53.3\%. Conclusions. The most used coping styles by patients were positives and focused to emotion.
\end{abstract}

Key words: Cervical cancer, gynecology. Peru. (Source DeCs BIREME).

Egresado de Enfermeria, Universidad Peruana Cayetano Heredia. Lima - Perú.

Licenciada en Enfemeria. Profesora Auxiliar, Facultad de Enfermería, Universidad Peruana Cayetano Heredia. Lima - Perú. 


\section{INTRODUCCIÓN}

En los últimos años, el número de mujeres con diagnóstico de cáncer de cuello uterino se ha ido incrementando, así lo demuestran las altas tasas de incidencia y mortalidad, en Latinoamérica y el Caribe; 30500 mujeres mueren anualmente por esta causa, correspondiendo la mayor proporción al grupo etario entre 35 a 54 años (1). En el Perú, el cáncer de cuello uterino es la primera causa de muerte en la población femenina en edad fértil, se ha estimado la incidencia de cáncer de cuello uterino en 40/100 000 (2). En el Instituto Nacional de Enfermedades Neoplásicas se atiende aproximadamente 1600 mujeres anualmente con esta enfermedad, para la mayoría de las pacientes con cáncer de cuello uterino es importante destacar los estilos conductuales que puedan emplear las pacientes a la hora de aceptar y convivir con la enfermedad (3).

El cáncer de cuello uterino se origina en las células que revisten el cuello uterino, es un proceso progresivo en el que las células normales cambian lentamente hasta transformarse en células precancerosas y, posteriormente, pueden llegar a convertirse en cáncer (4). Estos cambios se identifican mediante la prueba de Papanicolaou para prevenir el desarrollo de cáncer, este tipo de cáncer se contagia principalmente por medio del virus del papiloma humano (5).

El afrontamiento ante el cáncer es un proceso dirigido a manejar las demandas que implica el diagnóstico, el tratamiento y el seguimiento de la enfermedad. En este sentido, los estilos de afrontamiento ante el cáncer se emplean como un mecanismo que determinará la adaptación y la delegación de solución ante los problemas que surgen durante el proceso oncológico (6). El afrontamiento está constituido por estrategias dinámicas y cambiantes que se manifiestan por un conjunto de respuestas tales como: pensamientos, sentimientos, acciones que están dirigidas a la búsqueda o restablecimiento del equilibrio con el objetivo de resolver situaciones problemáticas y reducir las tensiones que ellas generan. Los estilos de afrontamiento son aquellas predisposiciones personales para hacer frente a diversas situaciones y son los que determinaran el uso de ciertas estrategias de afrontamiento, las cuales son los procesos concretos y específicos que se utilizan en cada contexto. Sin embargo, algunos autores no hacen diferencia entre estilos y estrategias de afrontamiento. Según Carver, los estilos de afrontamiento son tres: enfocados al problema, a la emoción y los estilos adicionales de afrontamiento, por ende, el presente estudio consideró la clasificación de Carver por ser la más objetiva y orientadora (7).
Las pacientes con cáncer de cuello uterino, al tener una enfermedad crónica , requieren el control de distintas áreas, como los regímenes terapéuticos, cambios en los estilos de vida, reorganización de tiempo, sentimientos y emociones, mantenimiento relaciones adecuadas con personas significativas, del equilibrio entre la necesidad de pedir ayuda y la autosuficiencia; asimismo, en relación al proceso de afrontamiento algunos autores manifiestan que las pacientes pueden, inicialmente, rechazar la enfermedad, no creer en el diagnóstico, o minimizar su gravedad, causando gran impacto psicológico y emocional por parte de la paciente, lo que repercute en aspectos psicoafectivos tales como: estrés, incertidumbre, miedo, entre otros $(8,9)$.

Actualmente, se espera que las pacientes con cáncer de cuello uterino puedan emplear dichos estilos para aprender a manejar nuevas circunstancias que generen estrés. Es por esta razón que los estilos de afrontamiento que utilicen cada una de las pacientes les servirán para hacer frente a situaciones complejas que condicionen estrés durante el proceso de su enfermedad.

En tal sentido, el profesional de enfermería debe ayudar a la paciente a identificar y fortalecer estilos de afrontamiento positivos frente a la enfermedad con la finalidad de favorecer su pronta recuperación; asimismo, se observó que algunas pacientes en el servicio de ginecología se mostraban preocupadas y manifestaban no saber qué hacer frente a su actual diagnóstico. La consideración de que las pacientes con cáncer de cuello uterino experimentan las mayores aflicciones emocionales, motivó a realizar la presente investigación. ¿Cuáles son los estilos de afrontamiento en pacientes con cáncer de cuello uterino?, el objetivo fue determinar los estilos de afrontamiento, el propósito de motivar al personal de enfermería a revisar el plan de cuidados brindado en relación al uso de estilos de afrontamiento positivos.

\section{MATERIAL Y MÉTODOS}

La investigación fue de tipo descriptivo de corte transversal, realizado en los meses de agosto-septiembre del 2012. Se desarrolló en un hospital nacional de enfermedades neoplásicas ubicado en el distrito de Surquillo del departamento de Lima, en el servicio de ginecología ubicado en el primer nivel del pabellón principal. La población estuvo conformada por 202 pacientes nuevas con diagnóstico de cáncer de cuello uterino, La muestra se estimó por muestreo aleatorio simple, y estuvo constituida por 150 mujeres que firmaron el consentimiento informado y se encontraban en aparente buen estado físico y psicoló- 
gico al momento del estudio. Se les aplicó el inventario COPE, diseñado por Carver, y modificado por Crespo y Cruzado, quedando como BRIEF COPE el cual refiere una confiabilidad de alfa de Cronbach entre 0,60 y 0,90. Para mayor confiabilidad las investigadoras realizaron una prueba piloto con una muestra de 25 pacientes que no formaron parte de la muestra obteniendo una consistencia interna alfa de Cronbach 0,73 siendo este valor aceptable; los datos fueron vaciados en una base de datos diseñados en Excel y los resultados analizados porcentualmente empleando el programa estadístico SPSS.

\section{RESULTADOS}

Tabla 1. Estilos de afrontamiento enfocados en el problema en pacientes con cáncer de cuello uterino. INEN. Lima 2012

\begin{tabular}{|c|c|c|c|c|c|c|c|c|c|c|}
\hline \multirow{2}{*}{ Estilos de afrontamiento } & \multicolumn{2}{|c|}{ Nunca } & \multicolumn{2}{|c|}{ A veces } & \multicolumn{2}{|c|}{ A menudo } & \multicolumn{2}{|c|}{ S iemp re } & \multicolumn{2}{|c|}{ Total } \\
\hline & $\mathbf{N}$ & $\%$ & $\mathbf{N}$ & $\%$ & $\mathbf{N}$ & $\%$ & $\mathbf{N}$ & $\%$ & $\mathbf{N}$ & $\%$ \\
\hline $\begin{array}{l}\text { Concentro mis esfuerzos en hacer algo } \\
\text { acerca de ello. }\end{array}$ & 0 & 0 & 20 & 13,3 & 97 & 64,7 & 33 & 22,0 & 150 & 100 \\
\hline $\begin{array}{l}\text { Intento proponer una estrategia sobre qué } \\
\text { hacer. }\end{array}$ & 1 & 0,7 & 39 & 26,0 & 97 & 64,7 & 13 & 8,7 & 150 & 100 \\
\hline $\begin{array}{l}\text { Me centro en hacer frente a este proble ma, } \\
\text { y si es necesario dejo otras cosas un poco } \\
\text { de lado. }\end{array}$ & 0 & 0 & 35 & 23,3 & 92 & 61,3 & 23 & 15,3 & 150 & 100 \\
\hline $\begin{array}{l}\text { Llevo a cabo una acción directa en torno al } \\
\text { problema. }\end{array}$ & 0 & 0 & 40 & 26,7 & 98 & 65,3 & 12 & 8,0 & 150 & 100 \\
\hline $\begin{array}{l}\text { Me aseguro de no empeorar las cosas por } \\
\text { actuar precipitadamente. }\end{array}$ & 12 & 8,0 & 110 & 73,3 & 25 & 16,7 & 3 & 2,0 & 150 & 100 \\
\hline Elaboro un plan de acción. & 0 & 0 & 19 & 12,7 & 110 & 73,3 & 21 & 14,0 & 150 & 100 \\
\hline $\begin{array}{l}\text { Intento firme mente evitar que otras cosas } \\
\text { interfieran con mis esfuerzos por hacer } \\
\text { frente a esto. }\end{array}$ & 1 & 0,7 & 64 & 42,7 & 67 & 44,7 & 18 & 12,0 & 150 & 100 \\
\hline $\begin{array}{l}\text { Me obligo a esperar el mo mento adecuado } \\
\text { para hacer algo. }\end{array}$ & 15 & 10,0 & 53 & 35,3 & 80 & 53,3 & 2 & 1,3 & 150 & 100 \\
\hline $\begin{array}{l}\text { Intento conseguir consejo de alguien sobre } \\
\text { qué hacer. }\end{array}$ & 11 & 7,3 & 48 & 32,0 & 73 & 48,7 & 18 & 12,0 & 150 & 100 \\
\hline $\begin{array}{l}\text { Pregunto a personas que han tenido } \\
\text { experiencias similares qué hicieron. }\end{array}$ & 7 & 4,7 & 47 & 31,3 & 79 & 52,7 & 17 & 11,3 & 150 & 100 \\
\hline
\end{tabular}

Tabla 2. Estilos de afrontamiento enfocados en la emoción en pacientes con cáncer de cuello uterino. INEN. Lima 2012

\begin{tabular}{|c|c|c|c|c|c|c|c|c|c|c|}
\hline \multirow{2}{*}{ Estilos de afrontamiento } & \multicolumn{2}{|c|}{ Nunca } & \multicolumn{2}{|c|}{ A veces } & \multicolumn{2}{|c|}{ A menudo } & \multicolumn{2}{|c|}{ Siempre } & \multicolumn{2}{|c|}{ Total } \\
\hline & $\mathbf{N}$ & $\%$ & $\mathbf{N}$ & $\%$ & $\mathbf{N}$ & $\%$ & $\mathbf{N}$ & $\%$ & $\mathbf{N}$ & $\%$ \\
\hline $\begin{array}{l}\text { Intento cons eguir a poyo emocional de amigos } \\
\text { y familiares. }\end{array}$ & 6 & 4 & 41 & 27 & 90 & 60 & 13 & 9 & 150 & 100 \\
\hline Hablo con alguien de cómo me siento. & 8 & 5 & 35 & 25 & 85 & 57 & 20 & 13 & 150 & 100 \\
\hline $\begin{array}{l}\text { Intento verlo de man era diferente para hacerlo } \\
\text { parecer más positivo. }\end{array}$ & 2 & 1 & 46 & 31 & 90 & 60 & 12 & 8 & 150 & 100 \\
\hline $\begin{array}{l}\text { Acep to que esto ha pasado y que se puede } \\
\text { cambiar. }\end{array}$ & 1 & 1 & 43 & 29 & 87 & 58 & 19 & 13 & 150 & 100 \\
\hline $\begin{array}{l}\text { Intento desarrollarme como persona como } \\
\text { resultado de la ex periencia. }\end{array}$ & 0 & 0 & 20 & 13 & 130 & 87 & 0 & 0 & 150 & 100 \\
\hline Aprend o a vivir con ello. & 0 & 0 & 50 & 33 & 73 & 49 & 27 & 18 & 150 & 100 \\
\hline Busco la ayuda de Dios. & 0 & 0 & 1 & 1 & 19 & 13 & 130 & 87 & 150 & 100 \\
\hline Intento encontrar alivio en la religión. & 1 & 1 & 3 & 2 & 20 & 13 & 126 & 84 & 150 & 100 \\
\hline Me altero y dejo que mis emociones afloren. & 10 & 7 & 91 & 61 & 48 & 32 & 1 & 1 & 150 & 100 \\
\hline $\begin{array}{l}\text { Siento mucho malestar emocional y termino } \\
\text { por expresar ese malestar. }\end{array}$ & 3 & 2 & 105 & 70 & 37 & 25 & 5 & 3 & 150 & 100 \\
\hline
\end{tabular}


Tabla 3. Estilos adicionales de afrontamiento en pacientes con cáncer de cuello uterino. INEN. Lima 2012

\begin{tabular}{|c|c|c|c|c|c|c|c|c|c|c|}
\hline \multirow{2}{*}{ Estilos de afrontamiento } & \multicolumn{2}{|c|}{ Nunca } & \multicolumn{2}{|c|}{ A veces } & \multicolumn{2}{|c|}{ A menudo } & \multicolumn{2}{|c|}{ Siempre } & \multicolumn{2}{|c|}{ Total } \\
\hline & $\mathbf{N}$ & $\%$ & $\mathbf{N}$ & $\%$ & $\mathbf{N}$ & $\%$ & $\mathbf{N}$ & $\%$ & $\mathbf{N}$ & $\%$ \\
\hline Sueño despierta con cosas diferentes a esta. & 15 & 10 & 110 & 73 & 22 & 15 & 3 & 2 & 150 & 100 \\
\hline Me río de la situación. & 93 & 62 & 54 & 36 & 3 & 2 & 0 & 0 & 150 & 100 \\
\hline Hago bromas de la situación. & 50 & 33 & 95 & 63 & 4 & 3 & 1 & 1 & 150 & 100 \\
\hline Me digo a mí misma "esto no es real". & 12 & 8 & 95 & 63 & 38 & 25 & 5 & 3 & 150 & 100 \\
\hline Actúo como si nu nca hubiera suced ido. & 5 & 3 & 76 & 51 & 55 & 37 & 14 & 9 & 150 & 100 \\
\hline $\begin{array}{l}\text { Me vuelco en el trabajo y en otras actividades } \\
\text { sustitutivas para apartar cosas de mi mente. }\end{array}$ & 8 & 5 & 32 & 21 & 98 & 65 & 12 & 8 & 150 & 100 \\
\hline $\begin{array}{l}\text { Admito que no puedo hacerle frente y dejo de } \\
\text { intentarlo. }\end{array}$ & 15 & 10 & 105 & 70 & 16 & 11 & 14 & 9 & 150 & 100 \\
\hline $\begin{array}{l}\text { Reduzco la cantidad de esfuerzo que pongo en } \\
\text { marcha para resolver el problema. }\end{array}$ & 20 & 13 & 118 & 79 & 8 & 5 & 4 & 3 & 150 & 100 \\
\hline $\begin{array}{l}\text { Bebo alcohol o tomo dro gas para pensar menos } \\
\text { en ello. }\end{array}$ & 150 & 100 & 0 & 0 & 0 & 0 & 0 & 0 & 150 & 100 \\
\hline $\begin{array}{l}\text { Utilizo alcohol o drogas para ayudarme a } \\
\text { superarlo. }\end{array}$ & 150 & 100 & 0 & 0 & 0 & 0 & 0 & 0 & 150 & 100 \\
\hline
\end{tabular}

\section{DISCUSIÓN}

Con el objetivo de determinar cuáles son los estilos de afrontamiento en pacientes con cáncer de cuello uterino, se realizó una investigación con una muestra de $150 \mathrm{mu}-$ jeres que tenían las siguientes características: $54,6 \%$ tenían entre 35 a 59 años y $45,4 \%$ tenían entre 18 a 35 años. El 50\% tenía grado de instrucción primaria, el 40,7\% secundaria y el $9,3 \%$ superior; $46 \%$ procedían de la sierra, el $33,3 \%$ de la selva y el $20,7 \%$ de la costa; se encontraron los siguientes resultados que se discuten a continuación.

Con respecto a los estilos de afrontamiento enfocados al problema, entre el 73,3 al 52,7\% utilizó a menudo estilos positivos (tabla 1). En relación al afrontamiento activo emplearon a menudo los siguientes estilos: «llevo a cabo una acción directa en torno al problema» en el 65,3\%; «concentro mis esfuerzos en hacer algo acerca de ello» e «intento proponer una estrategia sobre qué hacer» en el 64,7\%; «me centro en hacer frente a este problema, y si es necesario dejo otras cosas un poco de lado» en el 61,3\%. Emplearon a veces: «me aseguro de no empeorar las cosas por actuar precipitadamente» en el 73,3\%. Menores porcentajes fueron encontrados por Martínez y Ojeda (2011) en una muestra de 44 pacientes con cáncer de mama, quienes habían culminado el tratamiento satisfactoriamente, el 12,9\% utilizó el afrontamiento dirigido al problema (10). La diferencia en los resultados podrían estar en relación al número de la muestra con respecto al presente estudio; además, las pacientes del presente estudio habían sido informadas recientemente sobre su diagnóstico de cáncer de cuello uterino, es decir, se hallaban en una etapa inicial de adaptación, la cual se caracteriza porque las pacientes se encontraban en un posible estado de incredulidad porque tienen una enfermedad difícil de afrontar y el tratamiento es incierto; asimismo, les genera miedo e incertidumbre, más aun cuando afecta su rutina de trabajo habitual, tareas cotidianas e incluso cuando las pacientes tienen niños pequeños $(11,12)$.

Con respecto a la planificación de actividades, utilizaron a menudo el estilo de afrontamiento: «elaboro un plan de acción» en el 73,3\%; es decir, pensar cómo manejarse frente al estresor, organizando y planificando estrategias de acción, escogiendo y estableciendo cual será la mejor estrategia para manejar el problema; los resultados podrían indicar que las encuestadas han planificado adecuadamente sus actividades a realizar. Sin embargo, el 53,3\% utilizó a menudo el estilo negativo refrenar el afrontamiento: «me obligo a esperar el momento adecuado para hacer algo»; dicho estilo se refiere a la capacidad de esperar o postergar el afronte al estresor hasta que se presente la oportunidad apropiada para actuar sobre el problema, impidiendo realizar alguna acción prematuramente; no obstante, al esperar para tomar una decisión se puede correr el riesgo de que sea muy tarde (13). Esto podría estar en relación con la búsqueda de mayor información y adquirir empoderamiento, lo que les permite obtener la seguridad que la acción a realizar es la más acertada. Además, se utilizó a menudo búsqueda de apoyo social por razones instrumentales: «pregunto a personas que han tenido experiencias similares qué hicieron» en el $52,7 \%$, con la finalidad de buscar en otras personas consejo, asistencia, información y orientación con el fin de solucionar el problema. Los resultados darían a entender que las pacientes 
prefieren informarse sobre otras experiencias similares a su estado de salud y, al estar informadas sobre su terapia, les facilita minimizar el miedo innato e incertidumbres a lo desconocido que toda persona siente en cualquier momento de su vida al afrontar una situación nueva, permitiéndoles mejorar la actitud frente a un agente estresor.

Con respecto a los estilos de afrontamiento enfocados a la emoción, entre el 87 al 57\% utilizó a menudo estilos positivos (tabla 2). Entre los estilos de afrontamiento positivos enfocados a la emoción utilizaron la aceptación: «intento desarrollarme como persona como resultado de la experiencia» en el $87 \%$, y «acepto que esto ha pasado y se puede cambiar» en el 58\%. La aceptación consiste en admitir que la situación estresante existe y acontece, considerada como un hecho con el que se tendrá que convivir ya que es inmodificable en el tiempo (13). Estos resultados podrían hacer suponer que las pacientes han crecido como personas, aprendiendo a ganar experiencias, que les permitirá afrontar situaciones difíciles con responsabilidad. Con respecto a la búsqueda de apoyo social por razones emocionales, el mayor porcentaje optó por el estilo: «intento conseguir apoyo emocional de amigos y familiares» en el 60\%, y «hablo con alguien de cómo me siento» en el $57 \%$. Similares resultados fueron encontrados por Gómez M. en una muestra de 14 mujeres diagnosticadas de cáncer de cuello uterino, donde encontraron que el apoyo social brindado por personal de salud, familia, vecinos y amigos, emergió como una estrategia de afrontamiento importante durante la trayectoria de la enfermedad (14). Las pacientes en ambos estudios optaron por expresar sus sentimientos en familiares y amigos, por tener confianza y afinidad con ellos, posiblemente así se sientan reconfortadas al ser escuchadas y comprendidas recibiendo apoyo moral de sus seres queridos, lo que es de vital importancia durante el proceso de recuperación debido a que es saludable exteriorizar los sentimientos y buscar ayuda si es necesario para evitar la depresión (15).

La muestra estudiada empleó a menudo la reinterpretación positiva y el crecimiento: «intento verlo de manera diferente para hacerlo parecer más positivo», en el $60 \%$. Similares porcentajes encontraron Gaviria A. Vinaccia, en una muestra de 28 pacientes con diagnóstico de diferentes tipos de cáncer en tratamiento quimioterapéutico del Instituto de Medicáncer de Medellín (Colombia) la reinterpretación positiva; fue el estilo más utilizado, al igual que en el presente estudio (16). Menores porcentajes fueron obtenidos por Palacio C. Hernández J, en su estudio del año 2009, en pacientes diagnosticadas con cáncer de cérvix en relación al tratamiento, realizado en una muestra de 20 mujeres que aluden el tipo de afrontamiento reinterpretación positiva llamándolo espíritu de lucha en el $10 \%$ (17), los resultados podrían deberse a que el tamaño de la muestra es menor. En el presente estudio los resultados encontrados revelan que las pacientes se muestran con una actitud positiva frente a una situación inesperada, la cual supone un cambio importante en su vida, la persona evalúa el problema de manera positiva, reconociendo o atribuyendo cualidades favorables a la experiencia estresante en sí misma; es decir, rescatando lo favorable de la situación adversa y asumiéndola como un aprendizaje para el futuro.

Las pacientes del estudio utilizaron como estilo de afrontamiento: «busco la ayuda de Dios» en el $87 \%$ e «intento encontrar alivio en la religión» en el $84 \%$. Similares resultados fueron encontrados por Delgado A, Melgarejo G, (2005) en una muestra de 70 mujeres con cáncer de mama, el estilo más utilizado fue acudir a la religión en el 81,45\% (18); asimismo, Ortiz. M, Sanchez J. (2008) en una muestra de 26 pacientes de cáncer de pulmón, encontraron que los estilos de afrontamiento más utilizados por las pacientes fue la ayuda espiritual (19); como se puede observar en ambos estudios las pacientes utilizaron el acudir a la religión, lo que nos indicaría que cada persona puede tener diferentes necesidades espirituales y al refugiarse en las mismas se sienten reconfortadas emocional y espiritualmente, encontrando la paz y tranquilidad que se requiere. Por ende, la importancia de las creencias religiosas es un recurso potencial y activo en el proceso de tratamiento y / o recuperación de la enfermedad debido a que provee soporte emocional (20).

En relación al uso del estilo de afrontamiento negativo: centrarse en las emociones y liberarlas, las encuestadas utilizaron a veces: «siento mucho malestar emocional y termino por expresar ese malestar» en el $70 \%$, y «me altero y dejo que mis emociones afloren», en el 61\%, al centrarse en las emociones y liberarlas, las pacientes se focalizan en las consecuencias emocionales desagradables expresando abiertamente dichas emociones (13), las mismas suelen expresar sus emociones negativas, tales como la ira, fastidio, y enojo de manera impulsiva evidenciando que en ocasiones no están en la capacidad de poder controlarlas, sobre todo al inicio del tratamiento.

En relación a los estilos adicionales de afrontamiento, la muestra optó por estilos negativos de afrontamiento entre el 79 al 51\% (tabla 3), utilizó a veces el desenganche mental: «sueño despierta con cosas diferentes a esta» en el 73\%. Además, utilizó a menudo el estilo de afrontamiento positivo: «me vuelco en el trabajo y en otras activi- 
dades sustitutivas para apartar cosas de mi mente» en el $65 \%$, las pacientes recurren a una amplia diversidad de actividades que la distraigan de su evaluación de los aspectos conductuales del problema, o de la meta con la que el estresor está interfiriendo; dando a entender que las pacientes prefieren actividades sustitutivas como el trabajo, actividades distractoras en general, mantenerse ocupadas y así despejar la mente, con la finalidad de apartar pensamientos negativos que puedan influir en su tratamiento.

Asimismo, utilizaron a veces el humor: «hago bromas de la situación» en el $63 \%$, el cual consiste en valorar la situación estresante con sentido del humor, incluye hacer bromas al respecto. Esto podría indicar que las pacientes recurren al humor con respecto a su enfermedad, lo que facilita la buena predisposición de su estado de ánimo. También las pacientes emplearon a veces dos estilos negativos, el desenganche conductual: «reduzco la cantidad de esfuerzo que pongo en marcha para resolver el problema», en el 79\%, y «admito que no puedo hacerle frente y dejo de intentarlo», en el 70\%, el mismo que consiste en la disminución del esfuerzo conductual y cognitivo dirigido al estresor, dándose la paciente por vencida sin intentar alcanzar las metas con las que el estresor está interfiriendo, la cual refleja que las pacientes optaron por estilos de afrontamiento negativos, lo que podría indicar que, en ocasiones, sienten pena, su autoestima es baja, se sienten solas e incluso podrían llegar a aislarse. Esto podría deberse al escaso apoyo social, que induce pensamientos negativos, pesimistas, los mismos que interfieren no solo en la parte cognitiva sino también en la conductual conllevando a que se den por vencidas antes de intentar luchar, lo cual propicia un declive en el proceso de recuperación.

Las encuestadas aplicaron a veces la negación: «me digo a mí misma esto no es real» en el 63\% y «actúo como si nunca hubiera sucedido» en el 51\%; la negación se da cuando la persona se rehusa a creer que el estresor existe o se comporta como si el estresor fuera irreal o sencillamente no existente (13). Menores porcentajes fueron obtenidos por Palacio C. Hernández J, en pacientes diagnosticadas con cáncer de cérvix en relación al tratamiento, en una muestra de 20 mujeres, encontraron que el $5 \%$ de las pacientes utilizaron los estilos negativos de fatalismo y negación (17). Las pacientes utilizaron dichos estilos de afrontamiento, lo que daría a entender que tienen tendencia a negar su enfermedad; debido al impacto que produce el diagnóstico de cáncer, esta reacción impide a la persona aceptar la realidad, manifestándose el escaso interés sobre su estado de salud. Los estudios difieren posiblemente a que la muestra del estudio de Palacio C. es menor con respecto al presente estudio. Además, nunca se mencionó el uso de sustancias «bebo alcohol o tomo drogas para pensar menos en ello» y nunca «utilizo alcohol o drogas para ayudarme a superarlo» ambos en el $100 \%$. El uso de substancias radica en recurrir al consumo de alcohol o drogas para aminorar las consecuencias emocionales desagradables que producen el problema o situación estresante (13). Afortunadamente, todas las pacientes nunca optaron por el uso de estas substancias nocivas.

Como se evidencia a través de los resultados encontrados, los estilos de afrontamiento más empleados por las pacientes con cáncer de cuello uterino fueron los estilos positivos y enfocados a la emoción: busco la ayuda de Dios. En los enfocados al problema: elaboro un plan de acción; en el estilo adicional fue: me vuelco en el trabajo y en otras actividades sustitutivas para apartar cosa de mi mente.

\section{REFERENCIAS BIBLIOGRÁFICAS}

1. Portugal A. Agenda Salud. Rv [revista en Internet]. 2006 Enero-Marzo [citado 13 Feb 2012]; 25(1). Disponible en http://www.documentacion.edex.es/ docs/1504ISIage8.pdf.

2. El cáncer cervical en Perú 2008. [citado 9 Mar 2012].Disponible en: http://www.cervicusco.org/ spanish/cervical-cancer-peru.html.

3. Instituto Nacional de Enfermedades Neoplásicas. Estadísticas (Enero-Diciembre 2011). [citado $28 \mathrm{Mar}$ 2012]. Disponible en: http://www.inen.sld.pe/portal/ documentos/pdf/estadistica/estadisticas/ 10032011_ENERO_2011.pdf.

4. American cáncer Society. Resumen sobre cáncer de cuello uterino. 2013; Disponible en: http://www. cancer.org/acs/groups/cid/documents/webcontent/ 003043-pdf.pdf

5. Bazan F. Psso M. y Gutierrez C. Conocimientos, actitudes y prácticas sobre la prueba de Papanicolaou. An. Facmed. (Internet). 2007, Vol. 68, n. 1 (citado 16 Abr 2012), pp. 47-54. Disponible en: http:// www.scielo.org.pe/pdf/afm/v68n1/a06v68n1

6. Galindo O. Valadez L. Estilos de afrontamiento en pacientes con cáncer testicular en tratamiento y seguimiento; experiencia del Instituto Nacional de Cancerología de México. 2013 [citado 15 Ene 2013].Disponible en: http://www.elsevier.es/es/ revistas/gaceta-mexicana-oncologia-305/estilosafrontamiento-pacientes-cancer-testiculartratamiento-seguimiento-90024241-articulosoriginales-2011. 
7. Fundación Site Ground Joomla Templates.Estrategias de afrontamiento.2010. [citado 15 Abr 2012]. Disponible en: http://www.todoesmente.com/estrategias-deafrontamiento.html.

8. Ciencia y enfermería. Modelo estructural de enfermería de calidad de vida e incertidumbre frente a la enfermedad. 2013. [citado 20 Ene 2013]. Disponible en: http://www.scielo.cl/scielo.php?pid=S0717$95532006000100002 \&$ script=sci_arttext.

9. Enríquez M. Estrategias de afrontamiento psicológico en cáncer de seno. Rev Univ. salud. 2010 Enero Diciembre. [citado 21 Ene 2013];12(1). Disponible en: http://www.scielo.org.co/scielo.php?script= sci_arttext\&pid=S0124-71072010000100002 \&lng=es\&nrm=iso

10. Martínez C. Ojeda S. Afrontamiento en mujeres de cáncer de mama; 2011 [citado 10 Abr 2012]. Disponible en: http://www.upch.edu.pe/duiict/vs2005/ LeeIsisScript.Asp.

11. Proceso de adaptación: fases [citado 10 Abr 2012]. Disponible en: https://sites.google.com/site/reded argentina/las-etapas-de-adaptacion-a-unaenfermedad-cronica

12. Londoño Y. El proceso de adaptación en las mujeres con cáncer de mama. Rev. Portal de Revistas en Ciencias de la Salud. 2009 Marzo. [citado 12 Abr 2012];27(1). Disponible en: http://pesquisa. bvsalud. org/portal/resource/pt/lil-518280.

13. Cassaretto M. Relación entre las cinco grandes dimensiones de la personalidad y el afrontamiento en estudiantes preuniversitarios de Lima Metropolitana. Universidad Nacional Mayor De San Marcos. 2009.

14. Gómez M., Lagoueyte M. El apoyo social: estrategia para afrontar el cáncer de cérvix. [citado 2012 Mayo 15]. Disponible en: http://www.scielosp.org/pdf/csp/ v15s2/1283.pdf.

15. Web. FEFOC. Familia [citado 15 May 2012]. Disponible en: http://www.familiaycancer.org/doc. php?op=familia

16. Gaviria A. Vinaccia S. Calidad de vida relacionada con la salud, afrontamiento del estrés y emociones negativas en pacientes con cáncer en tratamiento quimioterapéutico. [citado 10 Dic 2012].Disponible en: http://pepsic.bvsalud.org/scielo.php?pid= S0123417X 2007000200004\&script=sci_arttext

17. Palacio C. Hernandez A. Estilos de afrontamiento utilizados por las pacientes diagnosticadas con cáncer de cérvix en relación al tratamiento, de la Clínica Oncólogos de Occidente S.A de la ciudad de Pereira en el año 2009. [citado 4 Abr 2012]. Disponible en: http://biblioteca.ucp.edu.co:8080/jspui/bitstream/ 10785/565/1/completo.pdf

18. Delgado A. Melgarejo G. Relación entre la estrategia de afrontamiento más utilizada por las mujeres con cáncer de mama y algunas características socio demográficas. Lima: Universidad Peruana Cayetano Heredia; 2005. [citado 10 Abr 2012]. Disponible en: http://www.upch.edu.pe/duiict/vs2005/LeeIsis Script.Asp.

19. Ortiz M., Domingo J., Estilos de afrontamiento y apoyo social: su relación con el estado emocional en pacientes de cáncer de pulmón. Sociedad chilena de psicología clínica. 2007. [citado 10 Abr 2012]. Disponible en: http://teps.cl/files/2011/06/Art-46.pdf

20. La espiritualidad en el tratamiento de cáncer. Instituto nacional de cáncer de salud de EE.UU. [citado 22 Jun 2012]; Disponible en: http://www.cancer.gov/ espanol/pdq/cuidados-medicos-apoyo/espiritualidad/ HealthProfessional/page3

\section{Correspondencia}

Elena Tam Phun

Miguel Baquero 251, Lima.

Telefóno: 4314731 - 4244731

Correo electrónico: elena.tam@upch.pe

Forma de citar este artículo: Baiocchi-Morales A, Lugo-Ostos E, Palomino-Sanchez N, Tam-Phun E. Estilos de afrontamiento en pacientes con cáncer de cuello uterino. Servicio de ginecología de un Instituto Nacional. Rev enferm Herediana. 2013;6(1):18-24. 